\title{
Lewy body dementia: myth or mystery?
}

\author{
*Janet Carter, BSc, NBChB, MA, MRCPsych, Wellcome Research Training Fellow, Department of Neuroscience \& Section of Old Age \\ Psychiatry, Raymond Levy, MBChB, PhD, DPM, FRCPsych, Professor of Old Age Psychiatry, Institute of Psychiatry, De Crespigny Park, \\ London SE5 8AF, England \\ * Correspondence
}

\section{Introduction}

Psychiatry is a medical discipline long on disorders and short on explanations. ${ }^{1}$ The current debate concerning Lewy Body Dementia is surely enough to confirm this verdict. Although reports only number 300 cases, its rise to prominence has been rapid, provoking heated exchange in the literature and leading more recently to a demand for a review of existing clinical (and histological) classification systems. ${ }^{2}$

Lewy body dementia (LBD) (for the sake of nosological neutrality since up to 20 different rubrics currently exist) has been variously hailed as a new form of dementia with a distinct clinicopathological profile, so called diffuse Lewy body disease (DLBD) or senile dementia of the Lewy body type (SDLT), a variant of Alzheimer's disease - the Lewy body variant of Alzheimer's disease LBV or as part of a spectrum of Lewy body disorders including Parkinson's disease.

What is the clinician to make of this nosological quagmire and has any advance really been made since the early reports of 1961 ?

It seems clear that LBD is not a new illness and that it represents improved neuropathological detection and a higher index of suspicion which have highlighted its presence. The research base, although small, has identified several important considerations which suggest that LBD should not be ignored.

Firstly, prevalence rates in several studies ${ }^{3.4}$ confirm that it is not uncommon.

Secondly, it seems related to two major neurodegenerative conditions, Alzheimer's and Parkinson's diseases.

Thirdly, in the face of mounting clinical and genetic evidence for the heterogeneity of Alzheimer's disease (perhaps accounted for, in part by LBD) and the contam- ination of existing research data, we require an urgent revision of the current classification system.

\section{Lewy bodies}

Lewy bodies (LBs) are eosinophilic intraneuronal inclusion bodies which are the pathological hallmark of a number of neurodegenerative conditions including Parkinson's disease. LBs consist of abnormal accumulations containing the protein ubiquitin and phosphorylated and non-phosphorylated neurofilaments, important components of the neuronal cytoskeleton. Neurofilaments play an important role in axonal transport and determination of axonal calibre. Transgenic animal studies demonstrate that abnormal neurofilament accumulation can lead to neuronal dysfunction and degeneration .

Over the years there has been increased recognition from postmortem studies that LBs also occur in the cerebral cortex in a substantial number of patients with dementia. The numerous rubrics mentioned above have arisen because of the differences between the clinical syndromes which have been described to date in association with cortical Lewy bodies and the diagnostic significance attached to them. Review of the literature suggests differences in the sampling frames from which patients have been drawn may be responsible.'

Despite the apparent nosological confusion, a consensus is arising that most research groups are probably referring to a similar condition characterised at the neuropathological level by subcortical and cortical LBs formation, with or without a variable amount of Alzheimer-type pathology, predominantly senile plaques, with only a minority of patients having sufficient neurofibrillary tangles to meet quantitative neuropathological criteria for $\mathrm{AD}$.

\section{EDITORAL BOARD}

Editor-in-Chief: Brian A. Lawlor (Dublin). Editors: Timothy Dinan (London), David King (Belfast). Deputy Editor: Brian O'Shea (Dublin). Associate Editors: Ken Brown (Belfast), Patricia Casey (Dublin), Anthony Clare (Dublin), Stephen Cooper (Belfast), Thomas Fahy (Galway), Michael Fitzgerald (Dublin), Michael Kelleher (Cork), Brian Leonard (Galway), Roy McClelland (Belfast), Aidan McGennis (Dublin), Ciaran O'Boyle (Dublin), Eadbhard O'Callaghan (Dublin), Art O'Connor (Dublin), Ethna O'Gorman (Belfast), Ian Pullen (Edinburgh), David Sheehan (Tampa), Philip Snaith (Leeds), Hugh Staunton (Dublin), John Waddington (Dublin), Richard Williams (Calgary). Statisical Editor: Leslie Daly (Dublin). Deputy Statistical Editor: Ronan Conroy (Dublin). 


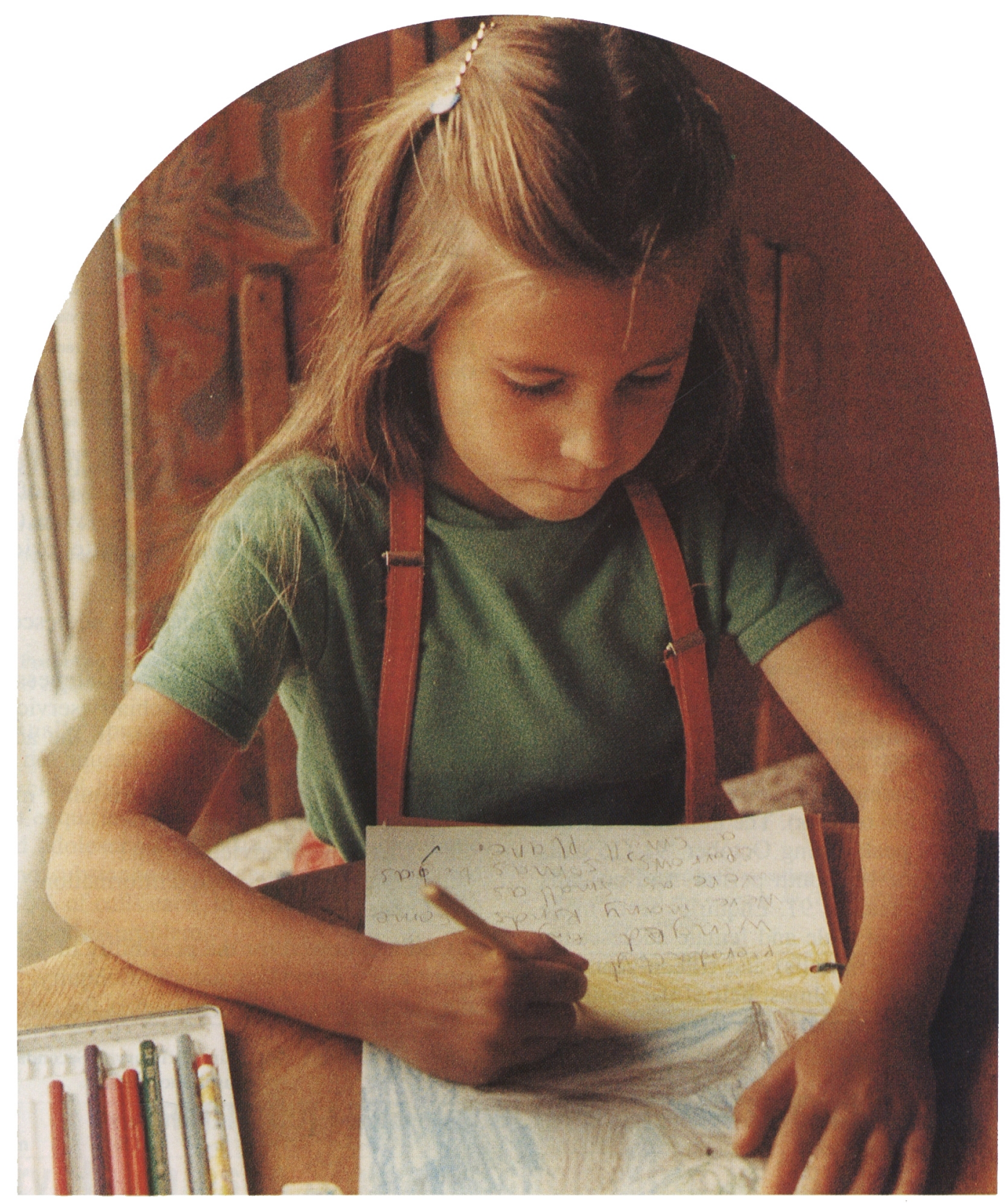

\section{Treats the Present and Safeguards the Future}

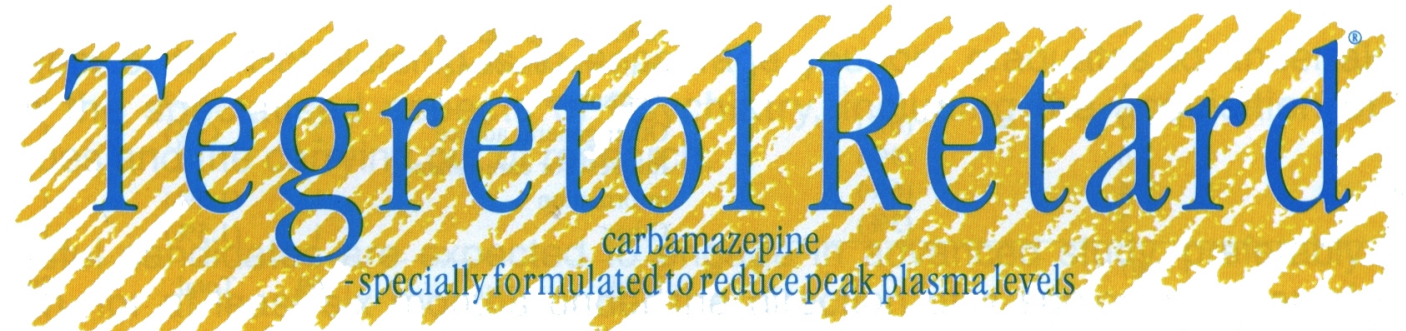

\section{For both generalised and partial seizures}

PRESCRIBING INFORMATION. Presentation: TEGRETOL RETARD is a formulation which reduces the peak concentration of active substance in the plasma and also ensures that fluctuations in plasma concentration are reduced throughout the day. Indications: Epilepsy (generalised tonicclonic and partial seizures). Trigeminal Neuralgia and other forms of deafferentation pain. Alcohol withdrawal symptoms. Treatment of mania and prophylaxis of manic-depressive illness Dosage: Epilepsy: Adults: $100-200 \mathrm{mg}$, once or twice daily, increasing slowly up to $800-1200 \mathrm{mg}$ dally, in divided doses. Children: See full prescribing information. Liquid is recommended for children under 5 years. It may be helpful to monitor drug levels: the optimum therapeutic plasma level RETARD should no be RETARD should not be chered, but swallowed whole with a inform information. Cont atrioventicula conduction abnimalities unless paced. Precautions: Blood counts should be performed before commencing treatment, at weekly intervals during the first month and subsequently monthly for five months then two to four times a year. Liver function tests should be performed before treatment and periodically during therapy. Withdraw TEGRETOL if allergic skin reactions, deterioration of liver function, or severe, progressive or clinically manifest leucopenia occur. Caution in patients taking oral anticoagulants or concomitant anti-epileptic or lithium therapy, or requiring oral contraception. Macrolide antibiotics (e.g. erythromycin), isoniazid, some calcium antagonists (i.e. Verapamil, diltiazem), a carbamazepine levels. CNS side-effects may be exacerbated by alcohol. TEGRETOL may impair reactions of patients driving or pering ant-conts during anti-convulsant therapy. See full prescribing information. IDE-EFFCrs: Occasionally dizziness, diplopia, headache, and agitation; dry mouth, nausea diarrhoea or constipation; loss of and agite; appetite; generalised erythematous rash; leucopenia, dermatitis, Stevens-Johnson Syndrome, toxic epidermal necrolysis, hair loss; proteinuria, lymph-node enlargement and acute renal failure; agranulocytosis, aplastic anaemia and thromboembolism (blood count should be checked regularly in early stages of treatment). Rarely dose-dependent hyponatraemia, disturbance to cardiac conduction, hepatitis. LEGAL CATEGORY: S1B. Pack of TEGRETOL RETARD divisible tablets of 200mg (PA 11/1/5) in blister packs of 100; TEGRETOL RETARD divisible tablets of 400mg (PA 11/1/6) in blister packs of 100. B) denotes Registered Trademark. Full prescribing information is available from Geigy Pharmaceuticals, Beech House, Beech Hill Office Campus PREPARATION February 1994. (C) Ciba Pharmaceuticals 1994

\section{Geigy}

Your Partner in Health Care 
The existence of some cases of LBD in the absence of any accompanying $\mathrm{AD}$ pathology and one study ${ }^{6}$ linking the number of cortical LBs to severity of dementia strengthen the case for the cortical LBs as a pathological substrate.

\section{Clinical evidence}

At the clinical level debate continues on whether there is actually an identifiable syndrome which distinguishes the dementia associated with Lewy bodies. Most clinical evidence against this position comes from studies in which patients diagnosed in life as having $\mathrm{AD}$, according to NINCDS-ADRDA criteria ${ }^{7,8}$ were found to have coincident LB pathology at postmortem.

Prospective examination of these subjects was unable to identify characteristic clinical features which might help to distinguish demented patients with LB from demented patients without LB. Protagonists have therefore argued that this proves the case for an $\mathrm{AD}$ variant. Others have argued that these cases are really misdiagnosed LBD and represent a small group of Lewy body patients who present with a "typical Alzheimer-type clinical picture". The NINCDS-ADRDA criteria would automatically exclude those with eg. Parkinson's disease or confusional states considered by current operational criteria to be central to the clinical picture.

In this context, it may be important to consider a major influence on clinical and histological classification systems for dementia. The early work of Tomlinson' has formed the basis for those currently in use. This emphasised the need to discriminate between $A D$ and vascular dementia, demonstrated in postmortem studies in the 1960 s to represent the two commonest causes of dementia in the elderly. Undoubtedly, this bias inherent in the current classification system accounts for some of the evidence which favours a Lewy body variant of AD.

\section{Diagnostic dilemma}

Operational criteria for Lewy body dementia ${ }^{10,11}$ represent the most rigorous attempts yet to define a clinical syndrome based on retrospective analysis of groups of neuropathologically proven cases of LBD. Systematic attempts to examine the reliability and validity of the criteria show that both have an acceptable reliability and the Newcastle criteria have a satisfactory sensitivity. ${ }^{12}$

If pathologically validated LBD patients are known to be erroneously diagnosed because they meet existing criteria for $\mathrm{AD}$ or vascular dementia where does this rather daunting picture leave the ordinary clinician? The operational criteria suggest that a typical LBD presents as a progressive dementia with fluctuating confusion, Parkinsonism and early, marked neuropsychiatric features, particularly visual hallucinations. This suggests an interplay of dual pathology with Parkinsonian features as an important pointer to the diagnosis.

Many features are shared with AD although psychiatric features are more common than in 'pure AD' and extrapyramidal features are milder than in classical PD. Pyramidal symptoms are rare and serve to distinguish
LBD from vascular dementia. However, patients who present with cortical symptoms which closely resemble the temporoparietal picture of typical AD present the greatest diagnostic challenge. Surely operational criteria as they undergo a process of refinement and adjustment must seek to identify these patients too?

\section{Genetics}

There are hints that molecular medicine may be able to shed some light on the above dilemmas. Taken in total, this evidence supports a clearer link with AD than with PD. For example, a single demented patient has been described from an amyloid precursor protein (APP) 717 mutation family who had both cortical LB and AD pathology, although this was not present in three other families with the same mutation. More compelling, however, is the finding ${ }^{13,14}$ that the frequency of the apoE4 allele, known to be associated with increased risk in familial and sporadic late onset $A D$, is similarly increased in patients with LBD but not in those with PD.

Cellular and transgenic models to determine the abnormality underlying neurofilament accumulation will help advance understanding at the molecular level still further.

\section{Implications for treatment}

Are the nosological disputes mentioned above merely academic or does current diagnostic inaccuracy have practical implications? This is perhaps best highlighted when considering options for pharmacotherapy. LBD patients will frequently have neuropsychiatric presentations and are consequently likely to attract intervention with neuroleptic medication. However, $60 \%$ of LBD patients have severe reactions to neuroleptic medication and the risk of mortality is increased two to three fold. ${ }^{15}$ Such interventions could prove highly dangerous in the LBD patient. Preliminary evidence suggests that the more selective dopamine antagonists including risperidone and clozapine may be less toxic.

Semi-quantitative analysis of L-Dopa treatment ${ }^{16}$ and an open trial of selegeline and L-Dopa ${ }^{17}$ in a small patient group suggest that there may be a place for such therapy particularly in those cases where symptoms early in the disease resemble classical Parkinson's disease.

However, anti-Parkinsonian drugs may exacerbate confusion, hallucinations and behavioural symptoms and a balance may have to be struck between lucidity and immobility.

Multicentre collaboration to study the neurochemistry of LBD compared to AD with cortical LB has demonstrated that irrespective of diagnosis LBs are associated with greater cholinergic deficits. This has given rise to speculation on the future place of anticholinesterase inhibitors in the treatment of these patients. Encouraging early anecdotal reports, however, do not seem to have been borne out by subsequent experience.

\section{Current theories}

Several possible explanations have been postulated to explain the overlap between AD pathology and subcorti- 
cal and cortical LB pathology. For example, it could be imagined that cortical and subcortical LB pathology lead to dementia when accompanied by chance by presymptomatic agerelated Alzheimer pathology. ${ }^{18}$ However, this seems less likely in view of recent quantitative evidence that AD pathology loading is greater in LB patients than in age matched normal controls. More in depth knowledge of the developmental sequence of pathology in $A D$ confirms that the $\mathrm{AD}$ pathology in $\mathrm{LB}$ patients is 'early stage' and not simply age related.

Perhaps a common biological factor, eg. apoE-4 genotype, predisposes to the formation of both types of cortical pathology. Alternatively, it could be that the total number of neuronal inclusions is important and LBs represent modified neurofibrillary tangles such that either may reflect a common aetiological process of $\mathrm{B}$ amyloid deposition. ${ }^{19}$

\section{Conclusion}

Dementia associated with cortical LB is the second commonest cause of cognitive impairment in the elderly. Although increased awareness has been the first stage in understanding, many fundamental questions remain unanswered. What underlies neurofilament accumulation? Are LBs important pathological substrates causally related to neuronal loss? How does LB pathology and coincident AD pathology relate and which is responsible for the clinical dementia? Current data are based on small samples and there is clearly a need to establish pathological criteria for LBD and careful prospective clinical studies drawn from as representative a population as possible. Perhaps answers from these future investigations will help unravel the mystery and dispel the myth.

\section{References}

1. McHugh PR. Witches, multiple personalities, and other psychiatric artifacts. Nature medicine 1995 110-5.

2. McKeith I, Fairburn AF, Perry RH, Thompson P. The clinical diagnosis and misdiagnosis of senile dementia of Lewy body type. Br J Psychiatry 1994; 165: 324-32.

3. Perry RH, Irving D, Blessed G, Perry EK, Fairburn AF. Senile dementia of Lewy body type: a clinically and neuropathologically distinct type of Lewy body dementia in the elderly. J Neurol Sci 1990; 95: 119-39.

4. Dickson DW, Farlo J, Davis P, Crystal H, Fuld P, Yen S-CH. A double labelling immunohistochemical study of senile plaques. Am J Path 1988; 132(i): 86101.

5. McKeith IG. Cortical Lewy Body Disease: the view from Newcastle. Developments in dementia and functional disorders in the elderly. London: Wrightson Biomedical Publishing Ltd, 1995. 6. Lennox G, Lowe J, Morrel K, Landon M, Mayer RJ. Diffuse Lewy body disease, correlative neuropathology using antiubiqutin immunocytochemistry. J Neurol Neurosurg Psych $1989 ; 52: 1236-47$

7. Hansen L, Salmon D, Galasko, Masliah E, Katzman $R$, De Teresa $R$, Thal L, Pay MM, Hofstestter R, Klauber M, Rice V, Butlers N, Alford $M$. The Lewy body variant of Alzheimer's disease. Neurology 1990; 40: 1-8.

8. Forstl H, Burns A, Luthert P, Cairns N, Levy R. The Lewy body variant of Alzheimer's disease: clinical and pathological findings. Br J Psychiatry 1993; 162: 385-92.

9. Thomlinson BE, Blesed G, Roth M. Observations on the brains of demented old people. J Neurol Sci $1970 ; 11: 205-42$.

10. Mc Keith IG, Fairburn AF, Perry RH, Jabeen S, Perry EK. Operational criteria for senile dementia of Lewy body type (SDLT). Psychol Med 1992; 22: 911-22.

11. Byrne EJ, Lennox G, Lowe J, Godwin RB, Jefferson D, Mayer RJ Landon M, Doherty FJ. Diagnostic criteria for dementia associated with cortical Lewy bodies. Dementia 1991; 2: 283-4.

12. McKeith IG, Fairburn AF, Bothwell RA, Moore PB, Ferrier IN, Thompson P, Perry RH. An evaluation of the predictive validity and inter-rater reliability of clinical diagnostic criteria for senile dementia of Lewy body type. Neurology 1994; 44: 872-7.

13. Benjamin R, Leake A, Edwardson JA, McKeith IG, Ince PG, Perry RH, Morris CM. ApoE allele frequencies in neurodegenerative disorders. Lancet 1994; 343: 1565.

14. Pickering-Brown S, Mann DMA, Bourke JP, Roberts DA, Bulderson D, Burns A, Byrne J, Owen F. Apoprotein E4 and Alzheimer's pathology in Lewy body disease and in other bamyloid forming diseases Lancet 1994; 343:1155

15. McKeith IG, Fairburn AF, Perry RH, Thompson $P$, Perry EK. Neuroleptic sensitivity in patients with senile dementia of Lewy body type BMJ 1992; 305: 673-8.

16. Byrne EJ, Lennox G, Lowe J, Godwin-Austen R. Diffuse Lewy body disease: clincal features in 15 cases. J Neurol Neurosurg Psychiatry 1989; 52: 709-17.

17. Williams SW, Byrne E;J, Stokes P. The treatment of diffuse Lewy body disease: a pilot study. Int J Ger Psychiatry; 8: 731-739

18. Quinn NP, Rossor MN, Marsden CD. Dementia and Parkinson's disease- pathological and neurological considerations. Br Med Bull 1986; 42: 86-90.

19. Gentleman SM, Williams B, Royston CM, Jagoe R, Clinton J, Perry RH, Ince PG, Allsop D, Polak JM, Roberts GW. Quantification of bA4 protein deposition in the medial temporal lobe: a comparison of Alzheimer's disease and senile dementia of Lewy body type. Neuroscience 1992; [letter]142: 9-12.

Original manuscript received March 15, 1995.

Final revision accepted August 10, 1995.

\section{Epilmo Prescribing Information (Eve)}

Presentation: 1. Epilim 200 Enteric Coated. A lilac-coloured, enteric coated tablet containing $200 \mathrm{mg}$ Sodium Valproate Ph Eur. 2. Epilim 500 Enteric Coated. A lilac-coloured, enteric coated tablet containing $500 \mathrm{mg}$ Sodium Valproate Ph Eur. 3. Epilim 100mg Crushable Tablets. A white, scored tablet containing $100 \mathrm{mg}$ Sodium Valproate Ph Eur. 4. Epilim Syrup. A red, cherryflavoured syrup containing 200mg Sodium Valproate Ph Eur. per $5 \mathrm{ml}$. 5. Epitim Liquid. A red, cherry-flavoured, sugar-free liquid containing $200 \mathrm{mg}$ Sodium Valproate Ph Eur. per $5 \mathrm{ml}$. 6. Epilim Intravenous. Off white sterile, freeze dried Sodium Valproate Ph Eur. $400 \mathrm{mg}$ in a clear glass vial supplied with an ampoule of $4 \mathrm{ml}$ of solvent (Water for Injections). Indications: In the treatment of generalised, partial or other epilepsies. Epilim Intravenous may be used for short term therapy, where oral treatment is temporarily not possible. Dosage and Administration: Daily dosage requirements vary according to age and bodyweight. Oral To be taken with or atter food. Epilim may be given twice daily. Enteric coated tablets should be swallowed whole. I.V. Epilim I.V should be reconstituted immediately prior to use and infusion solution containing it used within 24 hours. Any unused portion should be discarded. Epilim Intravenous may be given by direct slow intravenous injection or by infusion using a separate intravenous line in normal saline, dextrose $5 \%$, or dextrose saline. Monotherapy: Oral: Adults: Start at $600 \mathrm{mg}$ daily increasing by 200mg at 3-day intervals until control is achieved. (Maximum dose $2500 \mathrm{mg}$ per day]. Children over $20 \mathrm{~kg}$ : Initially $400 \mathrm{mg} /$ day with spaced increases until control is achieved. (Usually within the range $20-30 \mathrm{mg} / \mathrm{kg}$ bodyweight per day). Children under $20 \mathrm{~kg}: 20 \mathrm{mg} / \mathrm{kg}$ body weight per day: in severe cases may be increased but only when plasma valproic acid levels can be monitored. Above $40 \mathrm{mg} k \mathrm{~kg}$ per day monitor clinical chemistry and haematological parameters. IV.: Patients already satisfactorily treated with Epilim may be continued at their current dosage using continuous or repeated infusion: other patients may be given a stow intravenous injection over $3-5$ minutes, usually $400-800 \mathrm{mg}$ depending on bodwweight (up to $10 \mathrm{mg} / \mathrm{kg}$ ) followed by continuous or repeated infusion up to a maximum of $2500 \mathrm{mg} / \mathrm{day}$. Epilim Intravenous should be replaced by oral therapy as soon as practicable. Daily requirement for children is usually in the range $20.30 \mathrm{mg} / \mathrm{kg}$ day and method of administration is as above. Combined Therapy: It may be necessary to raise the dose when used with Combined Therapy: It may be necessary to raise the dose when used with anticonvulsants which induce live enzyme activity. Dosage of barbiturates
should be reduced if sedation is observed. Optimum dosage is mainly determined by seizure control and routine measurement of plasma levels is unnecessary. Contra-indications, Warnings, Etc. Contra-indications: Active liver disease, Family history of severe hepatic dysfunction, hypersensitivity to Sodium Valproate. Side-effects: Liver dysfunction including hepatic tailure resulting in fatalities has occursed in patients whose treatment included valproic acid or sodium valproate. Patients most at risk are children particularly those under the age of three and those with congenital metabolic disorders, organic brain disease or severe seizure disordess associated with mental retardation. The incidents mainly occurred during the first six months of therapy and usually involved multiple anticonvulsant therapy. Monotherapy is to be preferred in this group of patients. Clinical symptoms are more helpiul than laboratory investigations in the early stages of hepatic failure. Serious or fatal hepatotoxicity may be preceded by non-specific symptoms, usually of sudden onset, such as loss of seizure control, malaise, weakness, lethargy, oedema, anorexia, vomiting abdominal pain, drowsiness, jaundice. These are an indication for immediate withdrawal of the drug. Patients should be instructed to report any such signs to the clinician for investigations should they occur. Whilst it is difficult to establish which, if any, investigation is predictive, tests which reflect protein synthesis e.g. prothrombin time may be most relevant. Routine measurement of liver function should be undertaken before therapy and periodically during the first six months especially in those who seem most at risk, and those with a prior histon of liver disease. Hyperammonaemia without hepatic damage may occur; it is usually transient, but may occasionally present clinically. If so, Epilim should be discontinued. Valproic acid inhibits platelet aggregation. Thrombocytopenia has been reported. Prior to initiation of therapy and before surgery clinicians should assure themselves that there is no undue potential for bleeding complications. Spontaneous bruising or bleeding is an indication for withdrawal of medication. Pancreatitis, tremor increased appetite, weight gain, transient hair loss, increased alertness, aggressiveness, hyperactivity, irregular periods, amenorrhoea, gynaecomastia, stupor and oedema have been reported. Drug interactions Epilim has significant interactions with phenytoin, lamotrigine and other anticonvulsants. Epilim may potentiate the effects of neuroleptics, MAOls and other antidepressants, anticoagulants and salicylates. Cimetidine may inhibit the metabolism of Epilim. Epilim has appreciably less enzyme inducing effects than certain other anti-convulsants and loss of efficacy of oral contraceptive agents does not appear to be a problem. Women of Childbearing Age: An increased incidence of congenital abnormalities (including tacial dysmorphia, neural tube incidence of confenital abnormalities (including tacial dysmorphia, neural tube defects and multiple maliormations) has been demonstrated in offspring born to mothers with epilepsy both untreated and treated, including those treated with valproate during the first trimester has been estimated to be in the region of $1 \%$. Pregnancies should be carefully screened by alpha-fotoprotein measurement ultrasound and other techniques if appropriate. In all pregnancies monotherapy is to be recommended and dosape reviewed. The benefits of anti-epileptic therapy during pregnancy must be evaluated against the possible risks and therapy during pregnancy must be evaluated against the possible risks and patients should be informed of these and the need for screening. Folate has been
shown to reduce the incidence of neural tube defects in the offspring of high risk women in general although no direct evidence exists in relation to women receiving anti-epileptic drugs. However, there is no reason to contra-inidicate folic acid in these women. Product Authorisation Numbers: Evilim 200 Enteric Coated PA 77/113/1. Epilim 500 Enteric Coated PA 77/113/2. Epilim 100m Coated $77 / 3$. ED 77/113/6. Epilim I.V. PA 77/113/7. P.O.M. Date of Preparalion: February, 1995. Further information available on request from: Sanoti Winthrop Ireland Limited, United Drug House, Belgard Road, Tallaght, Dublin 24. Telephone: (01) 4621853/4621854. @ Registered trade mark.

Reterences:

1.Shovion S.D. The lancet, 1990; 336: 93-96.

2.Chadwick D.W., the Lancet, 1990; 336: 291-295.

3.Chadwick D.W., Epilepsia, 1987; 28 (2): $\$ 12.517$. 


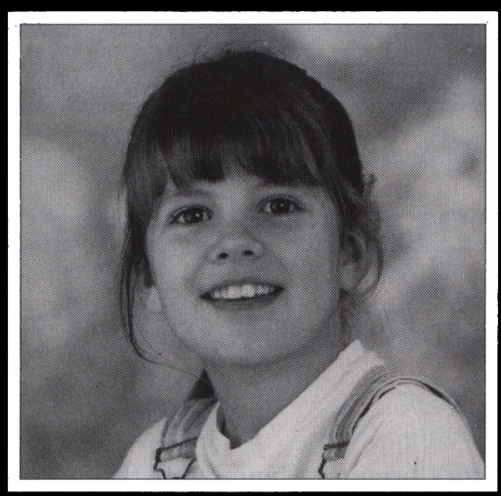

\section{Generalized}

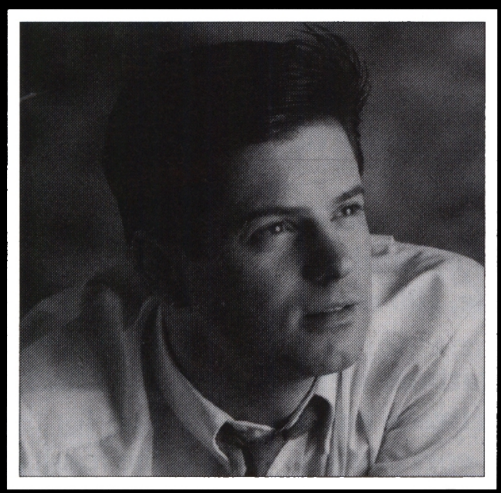

Partial

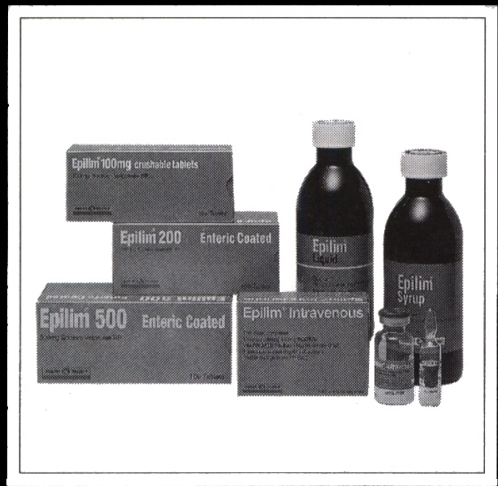

IMPARTIAL

Epilim means that you can treat generalized and partial seizures effectively, responsibly and confidently.

In fact, a 1990 survey in The Lancet has identified [Epilim] as a first-line anti-convulsant right across the range of epilepsies.'

Epilim also has a low incidence of serious side effects, ${ }^{2}$ minimal cognitive side effects, ${ }^{3}$ and a simple b.d. dosage regime which enhances compliance.

So, whichever type of epilepsy you're treating, you can be sure of one thing:

Epilim.

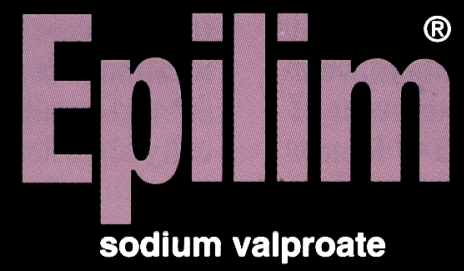

ESTABLISHED FIRST-LINE THERAPY ACROSS THE RANGE OF EPILEPSIES 